\title{
IL-7 and CCL19-secreting CAR-T cell therapy for tumors with positive glypican-3 or mesothelin
}

\author{
Nengzhi Pang ${ }^{1,4+}$, Jingxuan Shi ${ }^{2,3+}$, Le Qin ${ }^{2 \dagger}$, Aiming Chen ${ }^{5+}$, Yuou Tang ${ }^{1}$, Hainan Yang ${ }^{1}$, Yufeng Huang ${ }^{1}$, \\ Qingde Wu ${ }^{6}$, Xufeng Li ${ }^{1}$, Bingjia He ${ }^{1}$, Tianheng Li ${ }^{1}$, Baoxia Liang ${ }^{1}$, Jinglin Zhang ${ }^{1}$, Bihui Cao ${ }^{1}$, Manting Liu ${ }^{1}$, \\ Yunfei Feng ${ }^{1}$, Xiaodie Ye ${ }^{1}$, Xiaopei Chen ${ }^{1}$, Lu Wang ${ }^{1}$, Yu Tian ${ }^{1}$, Hao Li ${ }^{1}$, Junping $\mathrm{Li}^{1}$, Hong Hu${ }^{1}$, Jingping He${ }^{1}$, \\ Yuling $\mathrm{Hu}^{1}$, Cheng $\mathrm{Zhi}^{7}$, Zhaoyang Tang ${ }^{8,9}$, Yibo Gong ${ }^{10}$, Fangting $\mathrm{Xu}^{11}$, Linfeng $\mathrm{Xu}^{12}$, Weijun Fan ${ }^{13}$, \\ Ming Zhao ${ }^{13}$, Deji Chen ${ }^{1}$, Hui Lian ${ }^{1 *}$, Lili Yang ${ }^{4^{*}}$, Peng Li $^{2^{*}}$ (D) and Zhenfeng Zhang ${ }^{1^{*}}$
}

\begin{abstract}
Although chimeric antigen receptor (CAR)-engineered T cells have shown great success in the treatment of B cell malignancies, this strategy has limited efficacy in patients with solid tumors. In mouse CAR-T cells, IL-7 and CCL19 expression have been demonstrated to improve T cell infiltration and CAR-T cell survival in mouse tumors. Therefore, in the current study, we engineered human CAR-T cells to secrete human IL-7 and CCL19 $(7 \times 19)$ and found that these $7 \times 19$ CAR-T cells showed enhanced capacities of expansion and migration in vitro. Furthermore, $7 \times 19$ CAR-T cells showed superior tumor suppression ability compared to conventional CAR-T cells in xenografts of hepatocellular carcinoma (HCC) cell lines, primary HCC tissue samples and pancreatic carcinoma (PC) cell lines. We then initiated a phase 1 clinical trial in advanced HCC/PC/ovarian carcinoma (OC) patients with glypican-3 (GPC3) or mesothelin (MSLN) expression. In a patient with advanced HCC, anti-GPC3-7 $\times 19$ CAR-T treatment resulted in complete tumor disappearance 30 days post intratumor injection. In a patient with advanced PC, anti-MSLN-7 × 19 CAR-T treatment resulted in almost complete tumor disappearance 240 days post-intravenous infusion. Our results demonstrated that the incorporation of $7 \times 19$ into CAR-T cells significantly enhanced the antitumor activity against human solid tumor. Trial registration: NCT03198546. Registered 26 June 2017, https://clinicaltrials.gov/ct2/show/NCT03198546?term=NCT03 $198546 \&$ draw $=2 \&$ rank $=1$
\end{abstract}

\footnotetext{
*Correspondence: lian-hui-2008@163.com; yangll1@@mail.sysu.edu.cn;

li_peng@gibh.ac.cn; zhangzhf@gzhmu.edu.cn

${ }^{+}$Nengzhi Pang, Jingxuan Shi, Le Qin, Aiming Chen are contributed

equally to this work

${ }^{1}$ Department of Radiology; Translational Medicine Center

and Guangdong Provincial Education Department Key Laboratory of Nano-Immunoregulation Tumor Microenvironment, the Second Affiliated Hospital of Guangzhou Medical University, Guangzhou, Guangdong, China

${ }^{2}$ Key Laboratory of Regenerative Biology, Guangdong Provincial Key Laboratory of Stem Cell and Regenerative Medicine, Center for Cell Regeneration and Biotherapy, Guangzhou Institutes of Biomedicine and Health, Chinese Academy of Sciences, Guangzhou, China

${ }^{4}$ Department of Nutrition; Guangdong Provincial Key Laboratory of Food,

School of Public Health, Sun Yat-Sen University, Guangzhou, Guangdong, China

Full list of author information is available at the end of the article
} permits use, sharing, adaptation, distribution and reproduction in any medium or format, as long as you give appropriate credit to the original author(s) and the source, provide a link to the Creative Commons licence, and indicate if changes were made. The images or other third party material in this article are included in the article's Creative Commons licence, unless indicated otherwise in a credit line to the material. If material is not included in the article's Creative Commons licence and your intended use is not permitted by statutory regulation or exceeds the permitted use, you will need to obtain permission directly from the copyright holder. To view a copy of this licence, visit http://creativecommons.org/licenses/by/4.0/. The Creative Commons Public Domain Dedication waiver (http://creativeco mmons.org/publicdomain/zero/1.0/) applies to the data made available in this article, unless otherwise stated in a credit line to the data. 
Keywords: CAR-T cell, IL-7, CCL19, Glypican-3, Mesothelin, Pancreatic carcinoma, Hepatocellular carcinoma, Complete response

\section{To the editor:}

A recent study showed that IL-7 and CCL19 $(7 \times 19)$ secretion promotes the infiltration and survival of murine CAR-T cells in vivo [1-3]. To further verify the effects of $7 \times 19$ secretion on human CAR-T cells, we constructed three CAR vectors targeting GPC3, MSLN, and CD20 (Fig. 1a) [4-6], which all contained intracellular domains of CD28 and TLR2 [7-9]. As the cargo capacity of the lentiviral vector is limited, human IL-7 and CCL19 linked through the $2 \mathrm{~A}$ peptide were inserted into a separate lentiviral vector (Fig. 1a). We then cotransduced $\mathrm{T}$ cells with CAR and $7 \times 19$ lentiviruses (Fig. 1b). IL-7 and CCL19 were detected in the supernatant of $7 \times 19$ CAR-T cell cultures (Fig. 1c). $7 \times 19$ augmented the chemotaxis and expansion of CAR-T cells (Fig. 1d, 1e), but did not influence the cytotoxicity and cytokine secretion of CAR-T cells (Additional file 1: Fig. S1a, S1b, S1c).

We next adopted a GPC3 ${ }^{+}$HepG2 cell line-derived xenograft $(\mathrm{CDX})$ mouse model to assess the antitumor efficacy of anti-GPC3-7 $\times 19$ CAR-T cells. AntiGPC3-7 $\times 19$ CAR-T cells significantly suppressed HepG2 growth than anti-GPC3 CAR-T cells (Fig. 1f, 1g). In addition, the percentage of $\mathrm{T}$ cells in peripheral blood of anti-GPC3-7 $\times 19$ group were higher than those in the anti-GPC3 group (Fig. 1h, Additional file 1: Fig. S2a). There were more infiltrated $\mathrm{T}$ cells in the tumors of the anti-GPC3-7 $\times 19$ group, compared that in the antiGPC3 group (Additional file 1: Fig. S2b). Similarly, antiGPC3-7 $\times 19$ CAR-T cells displayed superior antitumor efficacy in a HCC patient-derived xenograft (PDX) mouse model, compared to anti-GPC3 CAR-T cells (Additional file 1: Fig. S3). Moreover, anti-MSLN-7 $\times 19$ CAR-T cells also displayed more potent antitumor capacity than anti-MSLN CAR-T cells in a MSLN ${ }^{+}$AsPC-1 CDX mouse model (Fig. 1i, 1j, 1k, Additional file 1: Fig. S4).

Based on the preclinical results, we conducted a phase I clinical trial in advanced hepatocellular carcinoma (HCC), pancreatic carcinoma (PC) and ovarian carcinoma (OC) patients with GPC3 or MSLN expression to further explore the clinical safety and feasibility of $7 \times 19$ CAR-T cell therapy. The protocol of this trial is described in the supplemental materials and is depicted schematically (Additional file 1: Fig. S5, S6). Patients were enrolled based on the patient inclusion and exclusion criteria (Additional file 1: Table S1). Six patients were administered anti-GPC3/anti-MSLN-7 $\times 19$ CAR-T cells (Additional file 1: Table S2).

Subject GD-G/M-001 is an advanced HCC patient with GPC3 expression (Additional file 1: Fig. S7a, S7b). Three metastatic lesions were chosen to perform computed tomography $(\mathrm{CT})$ guided intratumor injection of CAR-T cells. Though the sizes of two lung nodules did not change significantly 60 days after CAR-T cells injection (Fig. $2 \mathrm{a})$, the liver tumor lesion $(1.2 \times 1.3 \mathrm{~cm})$ shrunk significantly on day 10 and completely disappeared on day 32 post anti-GPC3-7 $\times 19$ CAR-T cells injection (Fig. $2 b$ ). The patient did not suffer any toxic effects and had staging evaluation revealing partial response (PR) according to standard Response Evaluation Criteria in Solid Tumors (RECIST) version 1.1 on CT.

Subject GD-G/M-005 is an advanced PC patient with MSLN expression (Additional file 1: Fig. S7f). This subject progressed with a local lymph node metastasis $(24 \times 33 \mathrm{~mm})$ (Fig. 2 c). Anti-MSLN-7 $\times 19$ CAR-T cells were first infused through hepatic artery with heavy fever on the night without cytokine release syndrome

\footnotetext{
(See figure on next page.)

Fig. $17 \times 19$ CAR-T cells showed enhanced antitumor activity in xenografts. a Schemes of a lentiviral vector of anti-GPC3 CAR, anti-MSLN CAR and anti-CD20 CAR. IL-7 and CCL19 $(7 \times 19)$ were linked through the 2A peptide, and truncated CD19 was used to indicate $7 \times 19$ expression. b The percentage of CAR and $7 \times 19$ expression on transduced human T cells was analyzed by flow cytometry. $\mathbf{c}$ A total of $1 \times 10^{6} \mathrm{~T}$ cells from each group were cultured in fresh media for $24 \mathrm{~h}$. The supernatant was collected, and the secretion of IL-7 and CCL19 was analyzed by ELISA. The anti-IL-7R antibody and anti-CCR7 antibody were used during culture. Open circle represents the values of individual well. Error bar denotes the S.D. $\mathbf{d}$ A total of $1 \times 10^{6}$ anti-GPC3-7 $\times 19$ CAR-T cells or anti-GPC3 CAR-T cells were cultured in the lower chamber of the Transwell plate for $24 \mathrm{~h}$, and then the anti-GPC3 CAR-T cells labeled with CSFE were cultured in the upper chamber for $5 \mathrm{~h}$. The responder T cells that migrated from the upper chamber to the lower chamber were enumerated by flow cytometry. Error bar denoted the S.D. and the results were compared with an unpaired t-test. ${ }^{*} P<0.05,{ }^{* *} P<0.01,{ }^{* * *} P<0.001$. e A total of $2 \times 10^{6}$ transduced T cells from each group were cultured in fresh media. The cell number was counted every two days. Error bar denotes the S.D. The results were compared with two-way ANOVA. ${ }^{*} P<0.05,{ }^{* * *} P<0.01,{ }^{* * * *} P<0.001$. f Tumor volume of HepG2 subcutaneously inoculated mice. Tumor volume $=\left(\right.$ length $\times$ width $\left.{ }^{2}\right) / 2$. g Tumor weight of HepG2 subcutaneously inoculated mice. $\mathbf{h}$ Percentage of T cells in peripheral blood of HepG2 subcutaneously inoculated mice. $\mathbf{i}$ Tumor volume of AsPC-1 subcutaneously inoculated mice. $\mathbf{j}$ Tumor weight of AsPC-1 subcutaneously inoculated mice. $\mathbf{k}$ Percentage of T cells in peripheral blood of AsPC-1 subcutaneously inoculated mice. $\mathbf{f}$, i Error bar denotes the S.D. The results were compared with two-way ANOVA. ${ }^{*} P<0.05,{ }^{* *} P<0.01,{ }^{* * *} P<0.001 . \mathbf{g}, \mathbf{h}, \mathbf{j}, \mathbf{k}$ The error bar denotes the SD. The results were compared with ordinary one-way ANOVA. ${ }^{*} P<0.05,{ }^{* *} P<0.01,{ }^{* * *} P<0.001$
} 
A GPC3-28T2z EF1 1 Anti-GPC3 scFv CD28 TLR2 CD37 eGFP MSLN-28T2z EF1 $\alpha$ Anti-MSLN scFv CD28 TLR2 CD37 CD20-28T2z EF1 $\alpha$ Anti-CD20 scFv CD28 TLR2 CD3Z - eGFP.

$7 \times 19$ EF1 $\mathrm{IL}-7$ CCL19 CD19t $\square \mathrm{CD} 28 \mathrm{TM} \square 2 \mathrm{~A}$

\section{D}

C
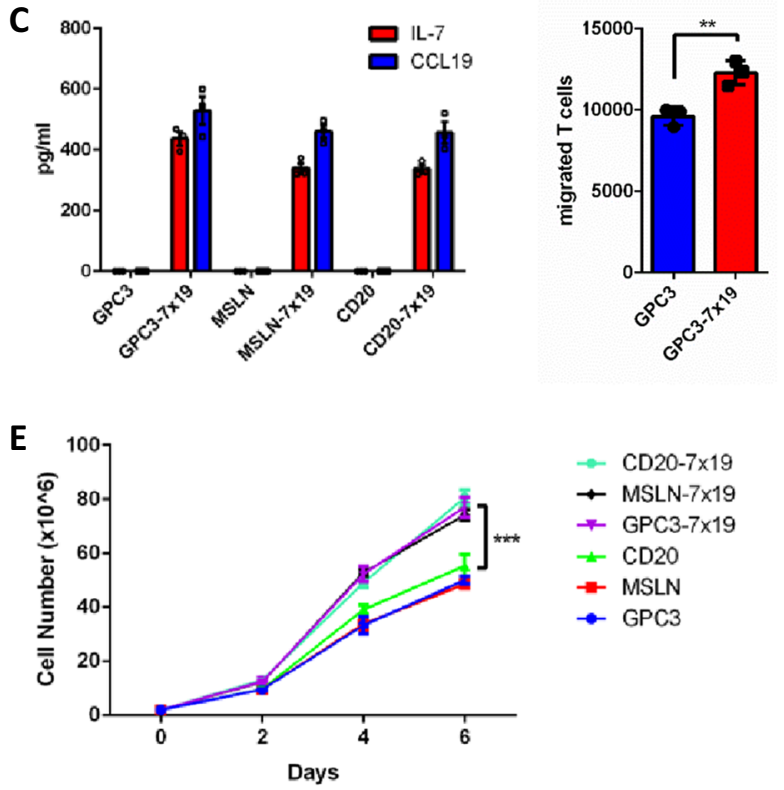

$\rightarrow$ CD20-7 $\times 19$

$\rightarrow$ MSLN-7 $7 \times 19$

- GPC3-7 $\times 19$

$-\operatorname{CD} 20$

- MSLN

$\rightarrow$ GPC3
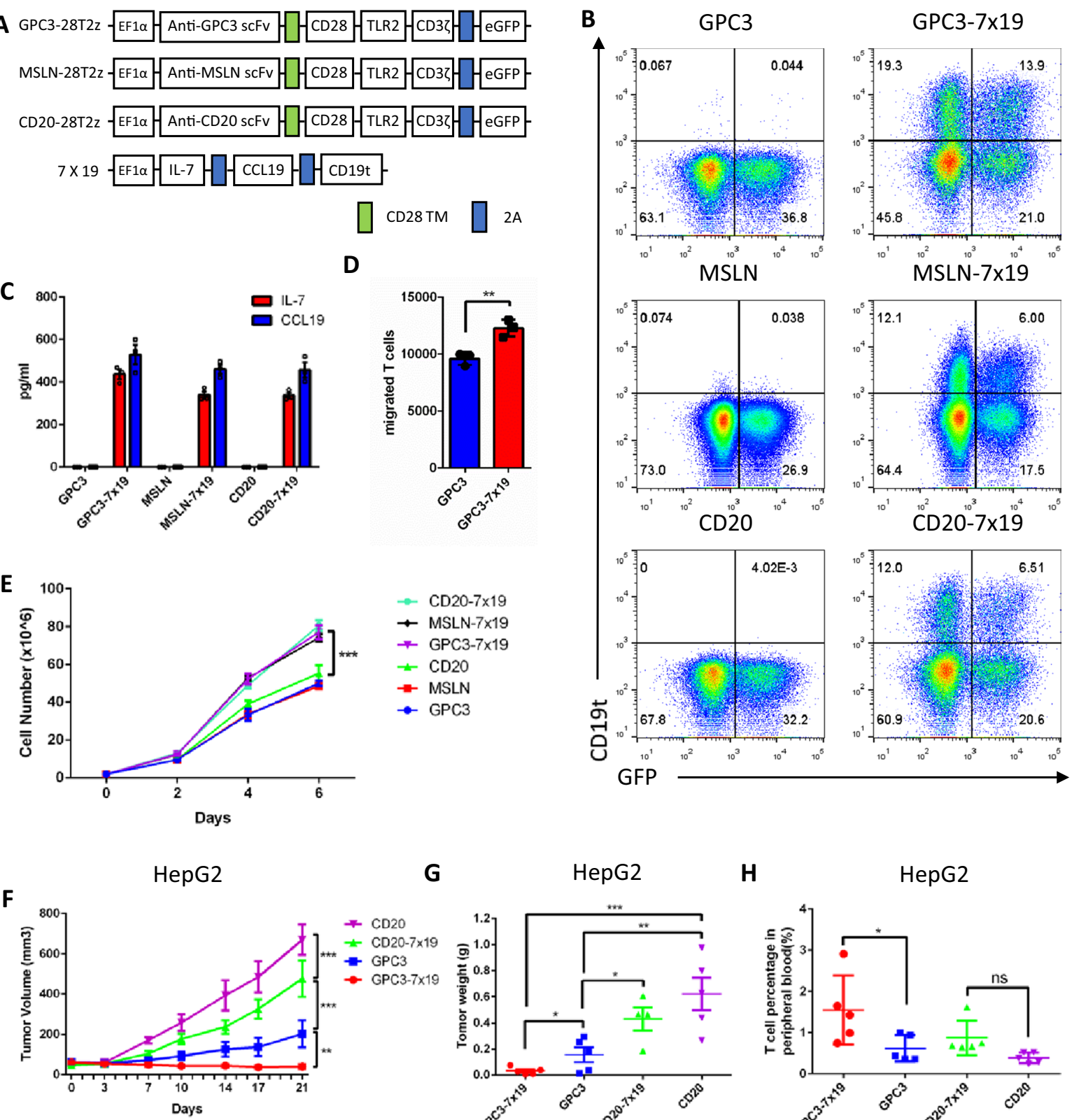

G

HepG2

H

HepG2
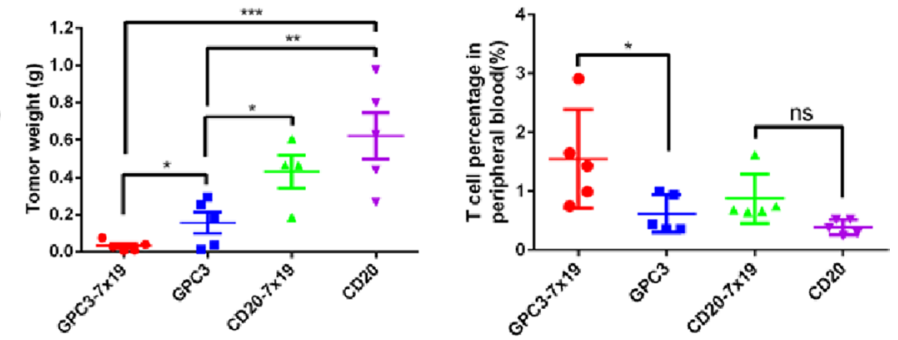

AsPC-1

J

AsPC-1

$\mathbf{K}$ AsPC-1
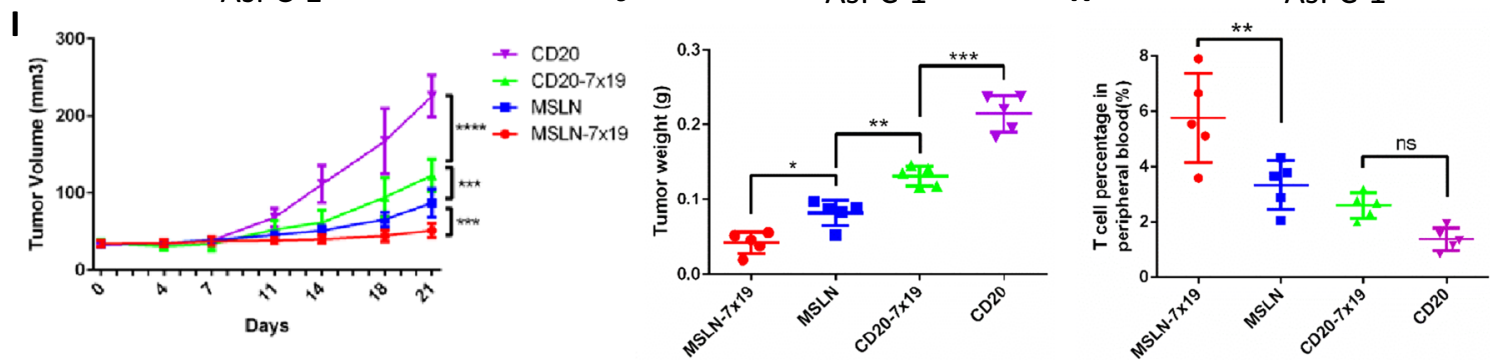

Fig. 1 (See legend on previous page.) 


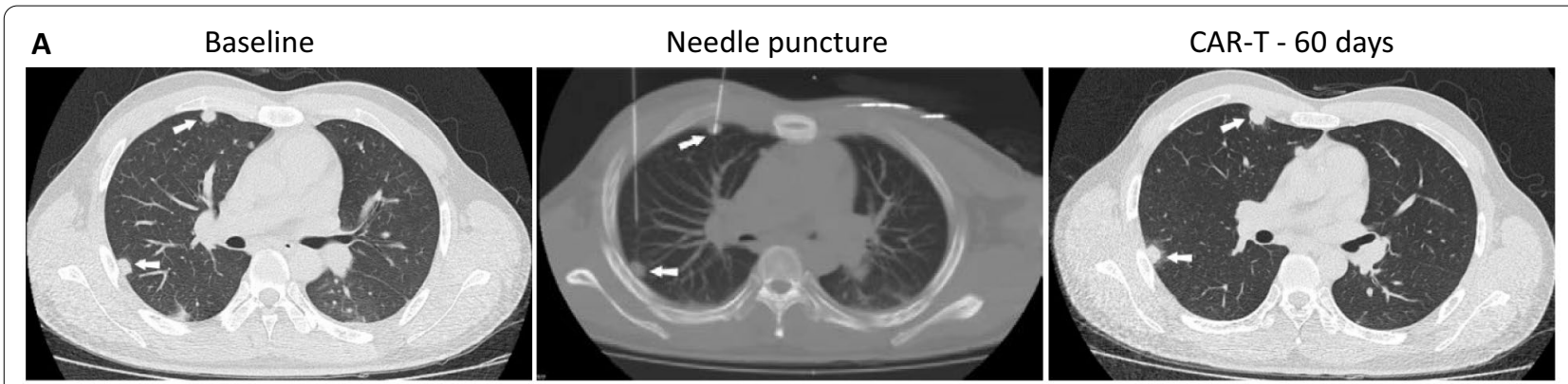

B

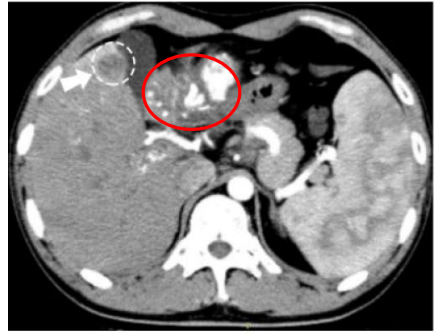

C

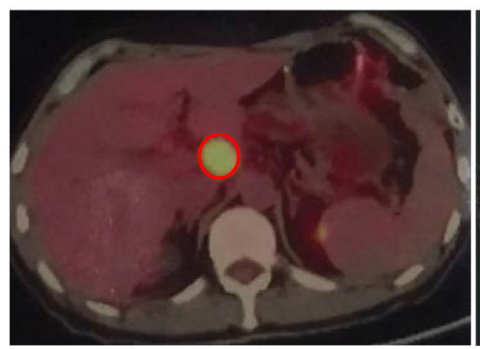

Needle puncture

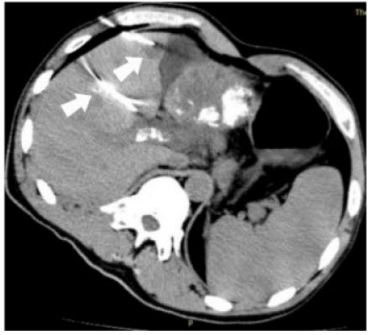

CAR-T - 10 days

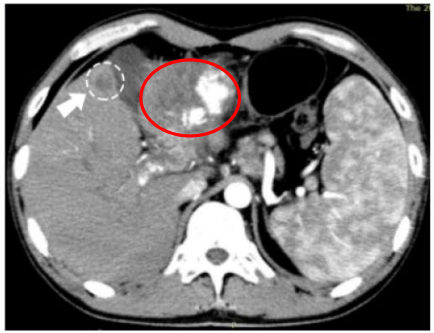

CAR-T - 32 days

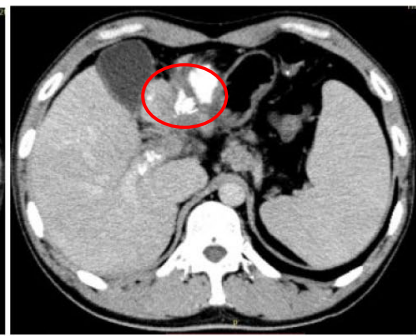

Fig. $27 \times 19$ CAR-T cells showed remarkable antitumor activity in HCC and PC patients with GPC3 and MSLN expression. For patient GD-G/M-001, three metastatic lesions were chosen to perform fine needles CT-guided intratumor injection of CAR-T cells. a One nodule $(0.5 \mathrm{~cm})$ in the right front lung, injected with anti-GPC3 CAR-T cells as a comparison; the second nodule $(0.5 \mathrm{~cm})$ in the right back lung, injected with anti-GPC3-7 $\times 19$ CAR-T cells. The results showed that the inhibited growth of anti-GPC3-7 $\times 19$ and anti-GPC3 CAR-T cells at similar levels 60 days after injection. Tumor is indicated by white arrow. $\mathbf{b}$ For the same patient GD-G/M-001, a tumor close to the gallbladder $(1.7 \times 2.0 \mathrm{~cm})$ was fine needle punctured under CT guidance and injected with anti-GPC3-7 × 19 CAR-T cells. 10- and 32-day follow-up CT scans showed a significant decrease in the size of the lesion and finally disappeared, respectively. At the same time, injection of the same CAR-T cells into the portal vein resulted in partial reopening of the previously obstructed portal vein. Metastatic tumor is indicated by white cycle; primary tumor is indicated by red cycle; white arrow points fine needle. c PET-CT and CT scans demonstrated a hepatic hilar lymph node metastasis after primary pancreatic carcinoma surgery in complete response in patient GD-G/M-005 following one intraartery and four intravein administrations of the autologous anti-MSLN-7 × 19 CAR-T cell infusion products. Tumor is indicated by red cycle

(CRS) or neurotoxicity [10]. Subsequently, he received intravenous infusions of the anti-MSLN-7 $\times 19$ CAR-T cells every 1-2 months. After 5 times of the CAR-T cells infusions, CT staging revealed complete response (CR) on day 240 with the lymph node measured $8.3 \times 9.6 \mathrm{~mm}$ and no other enlarged lymph nodes visible (Fig. 2c). He remained in normal condition.

The clinical course of the four other infused patients is shown in Additional file 1: Fig. S8 and described in Additional file 1: Table S3 and the Materials and Methods. Our current six-case cohort preliminary clinical study revealed no grade $2-4$ adverse events or major complications; one PC patient $(1 / 6,16.7 \%)$ achieved CR; one HCC patient $(1 / 6,16.7 \%)$ achieved PR; and 2 $(2 / 6,33.3 \%)$ achieved steady disease (SD), demonstrating the huge therapeutic potential of $7 \times 19$ CAR-T cell therapy for advanced solid tumors with GPC3/MSLN expression. Due to the limited number of cases enrolled in this study, we admit this was not a comprehensive clinical trial. However, based on the safety and efficacy of $7 \times 19$ CAR-T cells shown by these results, we are preparing to conduct a formal phase I clinical trials of 
$7 \times 19$ CAR-T cells that specifically target liver cancer or pancreatic cancer.

\section{Abbreviations}

CAR: Chimeric antigen receptor; human IL-7 and CCL19: 7 × 19; HCC: Hepatocellular carcinoma; PC: Pancreatic carcinoma; OC: Ovarian carcinoma; GPC3: Glypican-3; MSLN: Mesothelin; CR: Complete response; PR: Partial response: SD: Stable disease; PD: Progressive disease; CT: Computed tomography; RECIST: Response evaluation criteria in solid tumors; CRS: Cytokine release syndrome.

\section{Supplementary Information}

The online version contains supplementary material available at https://doi. org/10.1186/s13045-021-01128-9.

Additional file 1. Supplementary materials.

\section{Acknowledgements}

Not applicable.

\section{Authors' contributions}

Z.Z., P.L., D.C. and L.Y. jointly conceptualized and designed this study. N.P., J.S., L.Q. and H.L. wrote the manuscript, and Z.Z., P.L., D.C. and L.Y. participated in revising the article. Z.Z., D.C. and P.L. constructed the anti-GPC3-7 $\times 19$ CAR-T cells, and T.L., B.L. and J.Z. generated the CAR-T cell. J.S., N.P., B.C., Y.F., M.L. and H.H. performed the cell experiments. L.Q., X.C., L.W., Y.T., H.L., J.L., J.H., and $Y$. performed the animal experiments, including CDX and PDX mouse model establishment. B.L., J.Z., X.Y., Y.H., X.L., B.H. and T.L. prepared individual anti-GPC3-7 × 19 CAR-T cells for HCC patients, and Z.Z., D.C., A.C., H.L., Q.W., Y.G., F.X. and L.X. executed the interventional operation. X.Y., Y.T, H.Y., C.Z., Z.T., W.F., M.Z., L.Y., P.L., D.C. and Z.Z. collected and analyzed clinical data. All authors critically discussed the data and reviewed the final version of the manuscript. All authors read and approved the final manuscript.

\section{Funding}

This research was supported by the National Natural Science Foundation of China (No. 81672276 to Z. Z:; No. 81961128003 and 81972672 to P. L.; No. 81872613 and 81573142 to L. Y.; No. 81872069 to H. Y.), Guangzhou Science and Technology program (No. 201803010038 to Z. Z.); Strategic Priority Research Program of the Chinese Academy of Sciences (No. XDB19030205 and XDA12050305 to P. L.); The National Major Scientific and Technological Special Project for "Significant New Drugs Development (No. 2018ZX090201002-005); Guangdong provincial Significant New Drugs Development (No. 2019B020202003 to P. L.); Guangdong Special Support Program (No. 2017TX04R102 to P. L.); Guangzhou Science and Technology Plan Project (No. 201907010042 and 2020B1212060052 to P. L.); Frontier Research Program of Guangzhou Regenerative Medicine and Health Guangdong Laboratory (No. 2018GZR1 10105003 to P. L.); Science and Technology Planning Project of Guangdong province, China (No. 2017 B030314056 to P. L.); a grant from the University Grants Committee / Research Grants Council of the Hong Kong Special Administrative Region, China (No. AoE/M-401/20 to P. L.).

\section{Availability of data and materials}

All data are available in the manuscript or the supplementary materials.

\section{Declarations}

\section{Ethics approval and consent to participate}

This study was approved by the Human Ethics Committees of the Second Affiliated Hospital of Guangzhou Medical University, Guangzhou, China. The patient gave their written informed consent in accordance with the Declaration of Helsinki. This study is registered at www.ClinicalTrials.gov as NCT03198546. All animal experimental protocols were performed in accordance with instruction guidelines from the China Council on Animal Care and approved by the guidelines of the Ethics Committee of Animal Experiments at Guangzhou Institutes of Biomedicine and Health.

\section{Consent for publication}

The authors have obtained consent to publish from the participant to report individual patient data.

\section{Competing interests}

The authors declare no competing interests.

\section{Author details}

${ }^{1}$ Department of Radiology; Translational Medicine Center and Guangdong Provincial Education Department Key Laboratory of Nano-Immunoregulation Tumor Microenvironment, the Second Affiliated Hospital of Guangzhou Medical University, Guangzhou, Guangdong, China. ${ }^{2}$ Key Laboratory of Regenerative Biology, Guangdong Provincial Key Laboratory of Stem Cell and Regenerative Medicine, Center for Cell Regeneration and Biotherapy, Guangzhou Institutes of Biomedicine and Health, Chinese Academy of Sciences, Guangzhou, China. ${ }^{3}$ University of Chinese Academy of Science, Beijing 100049, China. ${ }^{4}$ Department of Nutrition; Guangdong Provincial Key Laboratory of Food, School of Public Health, Sun Yat-Sen University, Guangzhou, Guangdong, China. ${ }^{5}$ Department of Radiology, Qianjiang Central Hospital, Qianjiang, Hubei, China. ${ }^{6}$ Department of Radiology, Shunde Chinese Medicine Hospital, The Affiliated Hospital of Traditional Chinese Medicine University of Guangzhou, Foshan, China. ${ }^{7}$ Department of Pathology, The Second Affiliated Hospital of Guangzhou Medical University, Guangzhou, Guangdong, China. ${ }^{8}$ Guangdong Zhaotai Cell Biology Technology Ltd., Guangzhou, China. ${ }^{9}$ Guangdong Zhaotai InVivo Biomedicine Co. Ltd., Guangzhou, China. ${ }^{10}$ The Second Xiangya Hospital, Central South University, Changsha, China. ${ }^{11}$ Xiangya Hospital, Central South University, Changsha, China. ${ }^{12}$ Department of Interventional Radiology, Sun Yat-Sen Memorial Hospital, Sun Yat-Sen University, Guangzhou, China. ${ }^{13}$ Minimally Invasive Interventional Division; Department of Medical Imaging and Interventional Radiology; State Key Laboratory of Oncology in South China, Sun Yat-Sen University Cancer Center, Guangzhou, China.

Received: 26 May 2021 Accepted: 17 July 2021

Published online: 29 July 2021

\section{References}

1. Adachi K, Kano Y, Nagai T, Okuyama N, Sakoda Y, Tamada K. IL-7 and CCL19 expression in CAR-T cells improves immune cell infiltration and CAR-T cell survival in the tumor. Nat Biotechnol. 2018;36(4):346-51.

2. June $\mathrm{CH}, \mathrm{O}^{\prime} \mathrm{C}$ onnor RS, Kawalekar OU, Ghassemi S, Milone MC. CART cell immunotherapy for human cancer. Science. 2018:359(6382):1361-5.

3. Huang R, Li X, He Y, Zhu W, Gao L, Liu Y, Gao L, Wen Q, Zhong JF, Zhang C, Zhang X. Recent advances in CAR-T cell engineering. J Hematol Oncol. 2020;13(1):86

4. Wu AM, Tan GJ, Sherman MA, Clarke P, Olafsen T, Forman SJ, Raubitschek AA. Multimerization of a chimeric anti-CD20 single-chain Fv-Fc fusion protein is mediated through variable domain exchange. Protein Eng. 2001;14(12):1025-33.

5. Jiang Z, Jiang X, Chen S, Lai Y, Wei X, Li B, Lin S, Wang S, Wu Q, Liang Q, Liu Q, Peng M, Yu F, Weng J, Du X, Pei D, Liu P, Yao Y, Xue P, Li P. Anti-GPC3-CAR $T$ cells suppress the growth of tumor cells in patient-derived xenografts of hepatocellular carcinoma. Front Immunol. 2016;7:690.

6. Lv J, Zhao R, Wu D, Zheng D, Wu Z, Shi J, Wei X, Wu Q, Long Y, Lin S, Wang S, Wang Z, Li Y, Chen Y, He Q, Chen S, Yao H, Liu Z, Tang Z, Yao Y, Pei D, Liu P, Zhang X, Zhang Z, Cui S, Chen R, Li P. Mesothelin is a target of chimeric antigen receptor $T$ cells for treating gastric cancer. J Hematol Oncol. 2019;12(1):18.

7. Lai Y, Weng J, Wei X, Qin L, Lai P, Zhao R, Jiang Z, Li B, Lin S, Wang S, Wu Q, Tang Z, Liu P, Pei D, Yao Y, Du X, Li P. Toll-like receptor 2 costimulation potentiates the antitumor efficacy of CART Cells. Leukemia. 2018;32(3):801-8

8. Weng J, Lai P, Qin L, Lai Y, Jiang Z, Luo C, Huang X, Wu S, Shao D, Deng C, Huang L, Lu Z, Zhou M, Zeng L, Chen D, Wang Y, Chen X, Geng S, Robert W, Tang Z, He C, Li P, Du X. A novel generation 1928zT2 CART cells induce remission in extramedullary relapse of acute lymphoblastic leukemia. J Hematol Oncol. 2018;11(1):25. 
9. Liang Y, Liu H, Lu Z, Lei W, Zhang C, Li P, Liang A, Young KH, Qian W. CD19 CAR-T expressing PD-1/CD28 chimeric switch receptor as a salvage therapy for DLBCL patients treated with different CD19-directed CAR T-cell therapies. J Hematol Oncol. 2021;14(1):26.

10. Brudno JN, Kochenderfer JN. Toxicities of chimeric antigen receptor $T$ cells: recognition and management. Blood. 2016;127(26):3321-30.

\section{Publisher's Note}

Springer Nature remains neutral with regard to jurisdictional claims in published maps and institutional affiliations.
Ready to submit your research? Choose BMC and benefit from:

- fast, convenient online submission

- thorough peer review by experienced researchers in your field

- rapid publication on acceptance

- support for research data, including large and complex data types

- gold Open Access which fosters wider collaboration and increased citations

- maximum visibility for your research: over $100 \mathrm{M}$ website views per year

At BMC, research is always in progress.

Learn more biomedcentral.com/submissions 